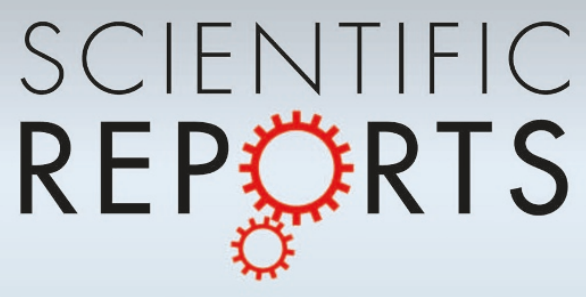

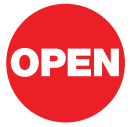

SUBJECT AREAS: ULTRAFAST PHOTONICS MATERIALS FOR OPTICS ELECTRICAL AND ELECTRONIC ENGINEERING

POLYMERS

Received

11 December 2012

Accepted

1 February 2013

Published

22 February 2013

Correspondence and requests for materials should be addressed to F.A. (francesco.arca. ext@siemens.com) or O.H. (oliver.hayden@ siemens.com)

\section{Interface Trap States in Organic Photodiodes}

\author{
Francesco Arca ${ }^{1,2}$, Sandro F. Tedde' , Maria Sramek' ${ }^{1}$ Julia Rauh ${ }^{3}$, Paolo Lugli² \& Oliver Hayden
}

\begin{abstract}
${ }^{1}$ Siemens AG, Corporate Technology, Günter-Scharowsky-Strasse 1, 91058 Erlangen, Germany, ${ }^{2}$ Institute for Nanoelectronics, Technical University of Munich, Arcisstrasse 21, 80333 Munich, Germany, ${ }^{3}$ Experimental Physics VI, Faculty of Physics and Astronomy, University of Würzburg, Am Hubland, 97074 Würzburg, Germany.
\end{abstract}

Organic semiconductors are attractive for optical sensing applications due to the effortless processing on large active area of several $\mathrm{cm}^{2}$, which is difficult to achieve with solid-state devices. However, compared to silicon photodiodes, sensitivity and dynamic behavior remain a major challenge with organic sensors. Here, we show that charge trapping phenomena deteriorate the bandwidth of organic photodiodes (OPDs) to a few $\mathrm{Hz}$ at low-light levels. We demonstrate that, despite the large OPD capacitances of $\sim 10 \mathrm{nF} \mathrm{cm}^{-2}$, a frequency response in the $\mathrm{kHz}$ regime can be achieved at light levels as low as $20 \mathrm{nW} \mathrm{cm}$-2 by appropriate interface engineering, which corresponds to a 1000-fold increase compared to state-of-the-art OPDs. Such device characteristics indicate that large active area OPDs are suitable for industrial sensing and even match medical requirements for single $\mathrm{X}$-ray pulse detection in the millisecond range.

$\gamma$ -ray radiation detection is a key application in medical diagnostics and requires highly sensitive detectors to ensure both high image quality and low patient dose. In general the detection is performed by indirect Xray conversion using a thin film scintillator material, such as terbium-doped gadolinium oxysulfide (GOS: $\mathrm{Tb}$ or GOS), to generate fluorescence at a wavelength that matches the spectral sensitivity of radiographic films or amorphous silicon (a-Si) photodiodes. Due to optic limitations to concentrate more X-ray photons into a small area, $\mathrm{X}$-ray photodetectors have to be large devices of several $\mathrm{cm}^{2}$ for sufficient sensitivity. In addition, X-ray detection for medical diagnostics requires high X-ray transparent devices. To reduce the total X-ray absorption of the detector the scintillator layer must be very thin. With typical dose rates such as applied in radiography the Xray conversion of the GOS thin film results in an extremely low green emission of a few $\mathrm{nW} \mathrm{cm}{ }^{-2}$ which requires highly sensitive detectors. Device sensitivity and response time needs to fit to today's digital X-ray imaging systems for medical diagnostics operating at low dose rates of $<1 \mathrm{mGy}$ at energies of typically $70 \mathrm{keV}$ with pulse lengths of $\sim 1 \mathrm{~ms}$. Such low bandwidths are easily accessible to crystalline Si photodiodes but remain challenging for OPDs on large area because of low charge carrier mobilities and high thin-film device capacitances of $\sim 10 \mathrm{nF}$ $\mathrm{cm}^{-2}$. Using organic photodetectors for X-ray applications would be beneficial due to their unique properties related to large active area processing possibilities, low temperature solution processing, and tailored spectral sensitivities ${ }^{1,2}$. Recent reports on organic imagers have demonstrated that non-structured OPDs on a-Si backplanes are attractive as alternative to pixelated a-Si PIN diodes due to the reduced lithography efforts ${ }^{1,2}$. In particular bulk heterojunction $(\mathrm{BHJ})^{3}$ based OPDs have shown high external quantum efficiency $(E Q E)$ due to the high absorption coefficient of the absorber and low dark current densities ${ }^{4}$ at field strengths $>10 \mathrm{~V} \mu \mathrm{m}^{-1}$. Cut-off frequency values of $\sim 1 \mathrm{MHz}^{5}$ for standard OPDs and $\sim 420 \mathrm{kHz}^{6}$ for inverted OPDs were reported, though these values were derived on small active area devices tested with pulsed light illumination in the $\mathrm{mW}$ $\mathrm{cm}^{-2}$ range, which is several orders of magnitudes higher than the light intensity detected for X-ray imaging. Our temporal response measurements in the range from $\mathrm{mW} \mathrm{cm}^{-2}$ to $\mathrm{nW} \mathrm{cm} \mathrm{cm}^{-2}$ show that cut-off frequencies of OPDs vary over five decades with the light intensity. Most recently, Tzabari et al. ${ }^{7}$ have discussed the influence of light intensities on solar cells efficiencies showing that the presence of traps in the $\mathrm{BHJ}$ cause enhanced recombination, an effect which is hidden at higher light intensities.

In our study we present the dynamic behavior of OPDs at different light levels varying the pulsed light illumination (50\% duty cycle) at visible wavelengths over four decades from $\sim 300 \mu \mathrm{W} \mathrm{cm}{ }^{-2}$ to $\sim 20 \mathrm{nW}$ $\mathrm{cm}^{-2}$. Our results show that stack interfaces rather than the bulk of the active layer have a major impact on the response speed of organic photosensors. Although interfaces are known to be a major parameter for the efficiency of organic electronic devices, such as organic LEDs ${ }^{8}$, interface effects on OPDs have not been studied thoroughly. Our model system is based on spray-coated OPDs which allow us to minimize the material consumption and offer at the same time the highest degree of freedom of the layer stack, obtaining superior device 
characteristics despite the device roughness ${ }^{4}$. We explore the influence of traps located at the interfaces between the BHJ and the electrodes. With appropriate and rather effortless interface engineering the response time of the OPDs can then be improved over three decades in the lower $\mathrm{nW} \mathrm{cm} \mathrm{cm}^{-2}$ regime. With such device characteristics the organic sensor can be used for the first time for single X-ray pulse detection with energies of $70 \mathrm{keV}$ and dose rates in the $\mathrm{nGy} \mathrm{s}^{-1}$ regime with millisecond resolution.

\section{Results}

Devices fabrication and characterization. The OPDs are based on a device stack with an anode, an interlayer (IL) as electron blocker, a $\mathrm{BHJ}$ layer and a cathode. Figure 1a shows the schematic layer stack illustrating the roughness of the spray-coated BHJ layer. Four different OPDs with different ILs are fabricated on a indium-tin-oxide (ITO) coated glass: first a diode with poly (3,4-ethylenedioxythiophene) poly (styrenesulfonate) (PEDOT:PSS) as common IL applied in solar cells and photosensors, second with poly (3-hexylthiophene) (P3HT) IL, third with a self-assembled monolayer (SAM) IL and last a diode without IL as reference. After deposition of the ILs a BHJ layer is spray-coated in ambient conditions from a solution of P3HT and $[6,6]$-phenyl $\mathrm{C}_{61}$ butyric acid methyl ester (PCBM). Al is thermally evaporated to form the top contact (see Methods for details). The BHJ thickness is selected in such a way that equal internal electric fields of all OPDs and thus comparable electrical characteristics are ensured. In addition, with $\mathrm{BHJ}$ thicknesses $>400 \mathrm{~nm}$ we have comparable internal quantum efficiencies in all devices. Current-voltage (IV) measurements of the four OPD stacks with an active area of $1 \mathrm{~cm}^{2}$

a

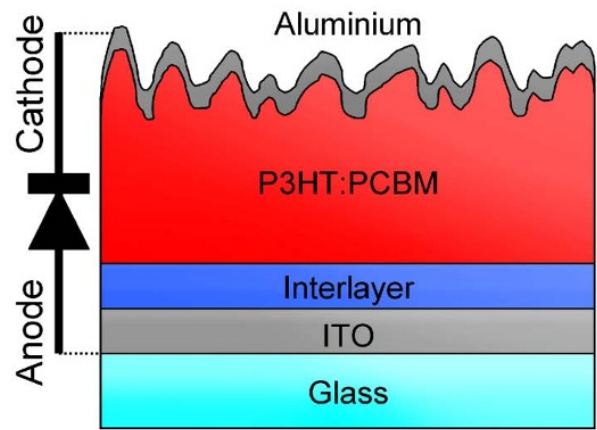

C

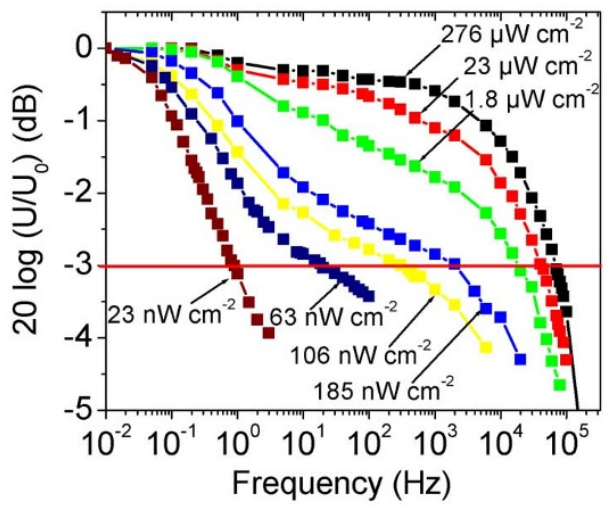

are shown in Figure 1b. Independent from the different ILs the diodes exhibit comparable low dark currents of $\sim 2 \times 10^{-5} \mathrm{~mA} \mathrm{~cm}^{-2}$ at $-5 \mathrm{~V}$ reverse bias and EQEs higher than $70 \%$ in the visible range (Supplementary Figure 1). Series resistance for all ILs measured at $1 \mathrm{~V}$ forward bias range from $250 \Omega$ to $1 \mathrm{k} \Omega$. The rectification ratio at $+/-1 \mathrm{~V}$ is the highest for the OPD with P3HT IL with $1.27 \times 10^{6}$ due to the lowest series resistance. All other devices have comparable rectification ratios of $2.9 \times 10^{5}$ (SAM IL), $1.6 \times 10^{5}$ (PEDOT:PSS IL), and $4 \times 10^{5}$ (without IL), respectively.

Dynamic response analysis. Figure 1c shows the dynamic measurements of the OPD with PEDOT:PSS as IL for various green light intensities, which is the reference diode in this study since PEDOT:PSS is the most commonly used IL for organic diodes. For high-light intensities of $\sim 276 \mu \mathrm{W} \mathrm{cm}$ cm $^{-2}$ at $532 \mathrm{~nm}$ wavelength the diode shows a cut-off frequency of about $71 \mathrm{kHz}$, comparable to reported values ${ }^{6}$. The cut-off frequency degrades to a few $\mathrm{Hz}$ when reducing the light intensity down to $20 \mathrm{nW} \mathrm{cm} \mathrm{cm}^{-2}$, i.e. varying the light intensity over four orders of magnitudes thereby a response time roll-off is observed over five orders of magnitudes. Similar dependencies of the cut-off frequency on the light intensity are observed with all other ILs, yet the low-light level response time differs significantly between devices with different IL which is not related to the serial resistance of the devices. The cut-off frequency dependency on the light intensity for all OPDs polarized at $-5 \mathrm{~V}$ is summarized in Figure 1d. PEDOT:PSS as well as P3HT are favorable as IL for high response time at light intensities $>200 \mathrm{nW} \mathrm{cm}^{-2}$, with cut-off frequencies in the $\mathrm{kHz}$ regime. However, below $200 \mathrm{nW} \mathrm{cm}^{-2}$

b

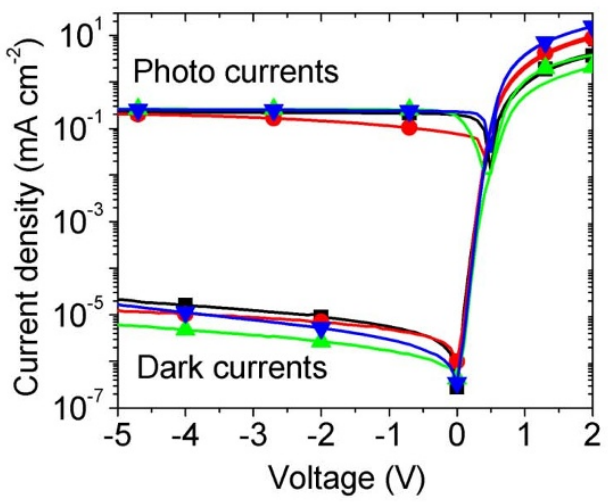

d

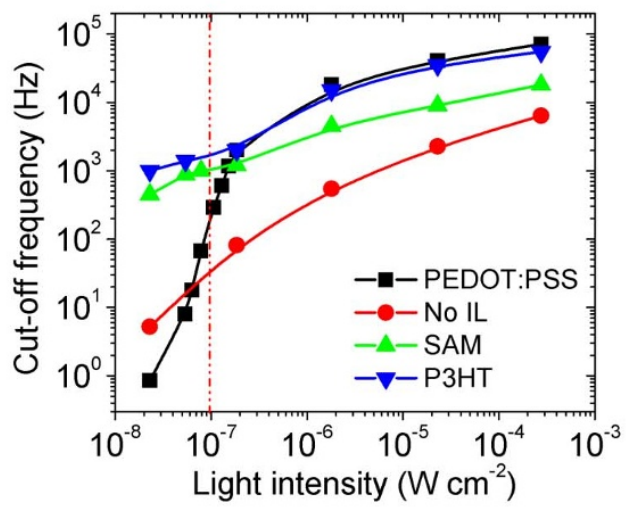

Figure $1 \mid$ Organic photodiode layout with $1 \mathrm{~cm}^{2}$ active area, electrical characteristics, and dynamic response with different ILs. (a) Layer stack of an OPD with ITO coated glass substrate, IL, spray-coated BHJ, and Al top electrode. (b) Dark and photocurrent characteristics of OPDs with PEDOT:PSS, P3HT and SAM ILs, and without IL (light illumination at $532 \mathrm{~nm}$ and $780 \mu \mathrm{W} \mathrm{cm}{ }^{-2}$ ). (c) Bode plot of a $-5 \mathrm{~V}$ reverse biased OPD with PEDOT:PSS IL with varying pulsed green light illumination ranging from $276 \mu \mathrm{W} \mathrm{cm} \mathrm{cm}^{-2}$ to $23 \mathrm{nW} \mathrm{cm}^{-2}$. (d) Response time dependence on light intensities of OPDs with PEDOT:PSS, P3HT and SAM IL, and without IL. 
the cut-off frequency of the device with PEDOT:PSS IL drops almost exponentially to a few $\mathrm{Hz}$ while the temporal response of the OPD with P3HT IL remains relatively stable in the $\mathrm{nW} \mathrm{cm}^{-2}$ regime. Devices with SAM IL always have a slightly lower cut-off frequency than OPDs with $\mathrm{P} 3 \mathrm{HT}$ IL with a relatively stable response time at illuminations $<200 \mathrm{nW} \mathrm{cm}^{-2}$. Devices without IL have the lowest response time throughout the observed light levels but show an even better characteristic than the reference OPD with PEDOT:PSS IL at intensities $<50 \mathrm{nW} \mathrm{cm}^{-2}$. Bode plot characteristics (Figure 1c) exemplary shown for a device with PEDOT:PSS IL as well as the crossing points of the cut-off frequencies of sensors with different ILs (Figure 1d) are significant and show a dramatic influence of the IL. Independent of the ILs the dynamic response of the OPDs is characterized by two different time constants at low and high frequencies. At high light illumination $\left(>1 \mu \mathrm{W} \mathrm{cm}{ }^{-2}\right.$ ) the pole at high frequency determines the $-3 \mathrm{db}$ cut-off frequency of the diode while under low light illumination $\left(<1 \mu \mathrm{W} \mathrm{cm}{ }^{-2}\right)$ the dynamic response is mainly given by the pole at low frequency (Figure 1c). No such pronounced effect on the diode speed is observed by varying cathode materials such as $\mathrm{Ca} / \mathrm{Ag}$ or $\mathrm{Al}$, as shown in Supplementary Figure 2. Furthermore, on a device with PEDOT:PSS IL varying the BHJ thickness by $30 \%$ has an insignificant effect on temporal characteristics of the diodes (cf. Supplementary Figure 3). Hence, the predominant factor in the variation of the cutoff frequency is given by the IL/semiconductor interface, which indicates a strong influence of IL/BHJ interface traps on the OPD dynamic response and can only be observed at low-light levels.

Thermally stimulated current analysis. To better understand the presence of interface traps and their respective energy levels a thermally stimulated current (TSC) analysis ${ }^{9,10}$ is performed on the same diodes. TSC spectra measured between $\sim 20 \mathrm{~K}$ and $\sim 250 \mathrm{~K}$ for OPDs with PEDOT:PSS and P3HT as IL and without IL are presented in Figure 2a. For the OPD with SAM IL, no TSC signal can be detected. This result is consistent with the high IL barrier (SAM is an insulator) which prevents carriers collection at low temperatures in the TSC experiment. The small current increase above $250 \mathrm{~K}$ for the OPD with PEDOT:PSS IL is not due to TSC and emptying of trap states, as it is also observed in the reference measurement without trap filling (data not shown) but can be attributed to the thermal injection of charge carriers by the electrodes. For all devices a TSC maximum between $90 \mathrm{~K}$ and $100 \mathrm{~K}$ is observed. The slight peak shifts in temperature probably originate from variations of the internal electric field, due to minor variations in film thicknesses and built-in voltages. Results on the OPD with PEDOT:PSS IL are in good agreement with literature ${ }^{11}$ (the small shift in the temperature can be attributed to the significantly different film thicknesses). Since both peaks show a similar shape, and in both studies the same active materials are used, the trap distribution is comparable. Schafferhans et al. ${ }^{11}$ calculate a broad trap distribution consisting of different trap levels with activation energies up to $\sim 0.4 \mathrm{eV}$ with a maximum at about $\sim 0.1 \mathrm{eV}$. However, the TSC peak of the OPD with PEDOT:PSS IL exhibits a more pronounced and longer high temperature tail compared to the reported solar cells, indicating a higher amount of deep traps at around $\sim 0.4 \mathrm{eV}$. These deep trap states are even more pronounced in the OPDs with P3HT IL and without IL (small TSC peak at $\sim 175 \mathrm{~K})$. Thus, two density maxima can be detected in the studied diodes at $\sim 0.1 \mathrm{eV}$ and $\sim 0.4 \mathrm{eV}$ above the P3HT HOMO level (Figure 2b). To gain deeper information about the exact trap distributions, especially of the deep traps, as well as on their origin (chemical, structural defects, impurities etc.) further investigations such as fractional TSC measurements or modulated photocurrent spectroscopy ${ }^{12}$ are required. It is important to mention that from the TSC only a lower limit of trap density could be obtained, since TSC signal is also given by incomplete trap filling, partial detrapping of charges during thermalization and recombination of the detrapped carriers with charges of the opposite signs. Amplitudes of the TSC signals are not relevant for the present analysis.

$\mathrm{X}$-ray results. With an OPD response time improved by 1000 -times and a respective cut-off frequency in the $\mathrm{kHz}$ regime at illumination levels of a few $\mathrm{nW} \mathrm{cm} \mathrm{cm}^{-2}$ we are able to perform single pulse X-ray detection by indirect energy conversion with a layer of GOS coupled to the bottom of the OPD substrate with transparent epoxy glue. The green fluorescence emission at $545 \mathrm{~nm}$ of the GOS film matches the highest spectral sensitivity of the photodiodes. GOS thin film thicknesses including voids were $\sim 12 \mu \mathrm{m}$ thick $(\sim 4 \mu \mathrm{m}$ absolute GOS thickness), which is sufficiently thin for shadow-free X-ray imaging at $70 \mathrm{keV}$ but thick enough for sufficient X-ray conversion with fluorescence emission intensities in the $\mathrm{nW} \mathrm{cm} \mathrm{cm}^{-2}$ regime. The influence of the ILs on the device response time is summarized in Figure 3a, where the normalized sensor response to a $2 \mathrm{~s}$ X-ray pulse of $90 \mu \mathrm{Gy} \mathrm{s}^{-1}(70 \mathrm{keV})$ of all OPD stacks is compared. Response times agree well with visible light measurements (cf. Figure 1d and 3a) with the fastest transients observed for P3HT and SAM ILs, whereas, PEDOT:PSS IL and OPD without IL show the longest transients. Direct X-ray conversion in the thin film $\mathrm{BHJ}$ devices can be neglected. In Figure $3 \mathrm{~b}$ the dynamic range of the X-ray sensor is shown with a linear correlation between dose rate and photocurrent from $\sim 3 \mu \mathrm{Gy} \mathrm{s}^{-1}$ up to $\sim 2.1 \mathrm{mGy} \mathrm{s}^{-1}$. The lowest detectable X-ray dose was $\sim 3 \mu \mathrm{Gy} \mathrm{s}^{-1}$ independent of the IL. Minimum rise and fall times of $\sim 1$ ms were observed with an OPD with P3HT IL reverse biased at $-5 \mathrm{~V}$ and irradiated with $420 \mu \mathrm{Gy} \mathrm{s}^{-1} \mathrm{X}$-ray train pulses with a length of $10 \mathrm{~ms}$ and a train period time of $50 \mathrm{~ms}$ (Figure $3 \mathrm{c}$ ). a

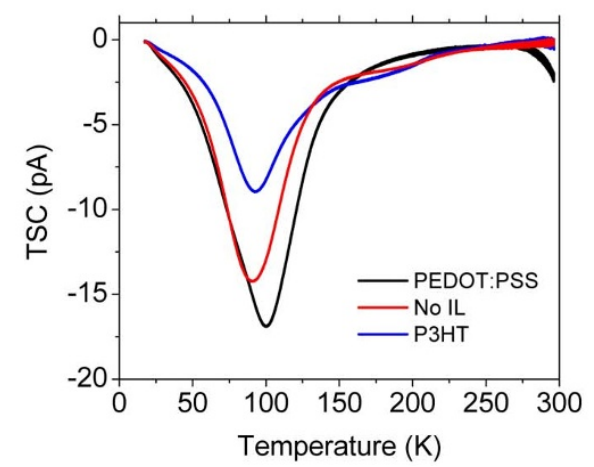

b

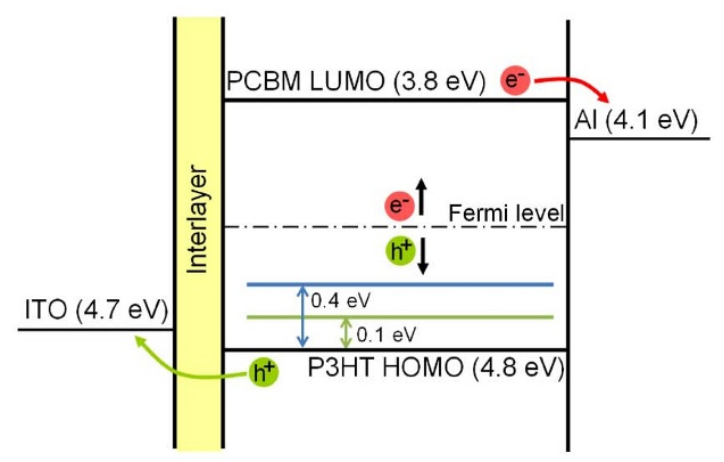

Figure $2 \mid$ Trap levels. (a) TSC measurements of OPDs without and with PEDOT:PSS or P3HT IL. (b) Flat band diagram model of an OPD. Two trap levels are positioned in the band gap. 
a

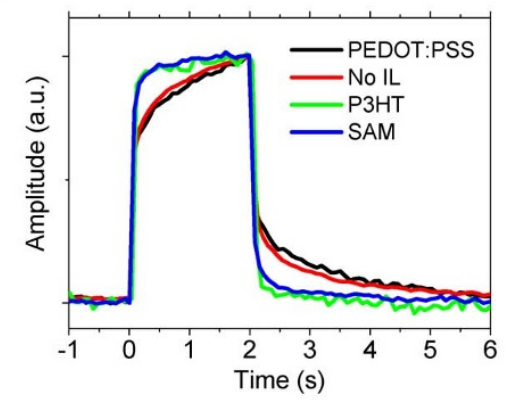

b

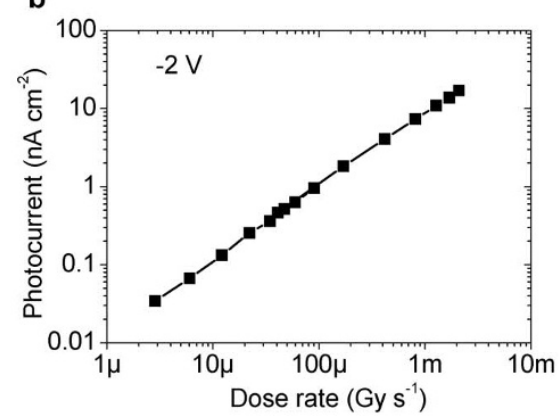

C

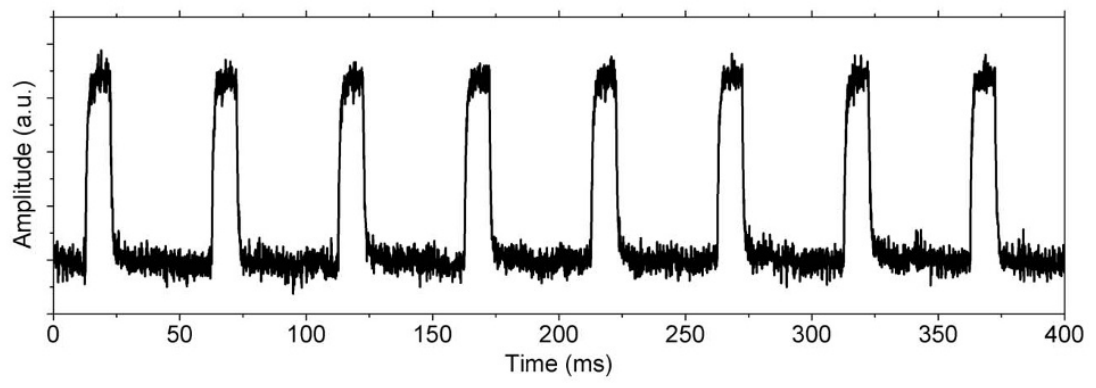

Figure $3 \mid$ IL influence on X-ray measurements with energies of $70 \mathrm{keV}$ and $12 \mu \mathrm{m}$ GOS thin film thickness for X-ray conversion.

(a) Sensor response of OPDs polarized at $-3 \mathrm{~V}$ with PEDOT:PSS, P3HT and SAM ILs and without IL to a single $90 \mu \mathrm{Gy} \mathrm{s} \mathrm{S}^{-1} \mathrm{X}$-ray pulse.

(b) Sensor response for the OPD with PEDOT:PSS IL reverse biased at $-2 \mathrm{~V}$ to X-ray pulses from $3 \mu \mathrm{Gy} \mathrm{s}^{-1}$ to $2.1 \mathrm{mGy} \mathrm{s}^{-1}$. (c) Sensor response of an OPD with P3HT IL reverse biased at $-5 \mathrm{~V}$ and irradiated by $70 \mathrm{keV}$ X-ray train pulses at $420 \mu \mathrm{Gy} \mathrm{s}^{-1}$ (10 ms pulse, $50 \mathrm{~ms}$ period length).

\section{Discussion}

Supplementary Table 1 summarizes our experimental observations and suggests the following charge transport phenomenon in the OPDs. At high light levels such as $780 \mu \mathrm{W} \mathrm{cm} \mathrm{cm}^{-2}$ the density of the photons hitting the surface is $\sim 2 \times 10^{15}$ photons $\mathrm{cm}^{-2} \mathrm{~s}$. A large number of charge carriers are generated in excess of the trap states at the interface. Hence, interface traps can be filled without any visible degradation of the photocurrent. The device response time is therefore dominated by a capture and emission process and by the time constant of the volume traps. In strong contrast, at very low-light levels such as $20 \mathrm{nW} \mathrm{cm} \mathrm{cm}^{-2}$, corresponding to an incident photon density of $\sim 5 \times 10^{10}$ photons $\mathrm{cm}^{-2}$ s only few charge carriers are generated and the time constant of the interface traps determines the cut-off frequency of the device. Introducing a new IL to replace the PEDOT:PSS apparently changes the trap emission and capture rate at the interface.

Hence, with all three ILs but SAM IL we observe the same trap energy levels. Even if the trap energy levels are similar for the different devices the trapping and de-trapping time constant in the OPD stacks must be substantially different. In brief, at low-light levels traps are not fully filled and their occupation change over time. Due to the IL properties the hole extraction efficiency will result in a modified dynamic response of the OPD over three orders of magnitudes.

In summary, our experimental results show that defects at the IL/ $\mathrm{BHJ}$ interface dominate the organic diode dynamic behavior at very low light intensities of $<100 \mathrm{nW} / \mathrm{cm}^{2}$. Such a major influence of the device speed can only be observed with high performance OPDs having both dark current densities $\sim 1 \times 10^{-5} \mathrm{~mA} \mathrm{~cm}^{-2}$ and high EQE of $\sim 70 \%$. With an optimized choice of the interlayer material only within the layer stack such as P3HT or a SAM three decades of response time can be gained without altering the IV characteristics. The devices match for the first time requirements for medical X-ray imaging. We propose that with further improvement of the IL an even higher dynamic response at ultra-low light levels can be achieved. Further potential can be envisioned in industrial process monitoring, where large-area low-cost organic photodetectors could replace conventional solid-state solutions.

\section{Methods}

Device fabrication. Four different OPDs with different ILs were fabricated on a $5 \times$ $5 \mathrm{~cm}^{2}$ structured ITO coated glass. In order to lower the dark currents ITO electrode rims were passivated by $\sim 1 \mu \mathrm{m} \mathrm{SU}-8$ transparent photoresist and a photoactive area of $1 \mathrm{~cm}^{2}$ of the anode defined by photolithography. Photoresist hard baking has been performed at $200^{\circ} \mathrm{C}$ for $15 \mathrm{~min}$ on a hotplate. After cleaning the substrates in acetone, isopropanol and deionized water RIE plasma was applied to activate the ITO before the deposition of the electron blocking ILs. First, a $\sim 150 \mathrm{~nm}$ PEDOT:PSS HIL 3.3N from HC Starck was spin-coated on ITO and baked in a vacuum oven at $200^{\circ} \mathrm{C}$ for $15 \mathrm{~min}$. Second, a P3HT layer from Rieke spray-coated on the ITO from a solution of $1 \% \mathrm{wt}$. in xylene resulting in a film thickness of $\sim 70 \mathrm{~nm}$. Third, a SAM formed by immersing the ITO in a 1\% wt. octadecylphosphonic acid solution in isopropanol until a contact angle $>110^{\circ}$ is measured. Last, a substrate without IL was applied as reference. After deposition of the ILs a BHJ layer was spray-coated in ambient conditions from a xylene solution of P3HT and PCBM with a weight ratio of $1: 0.75$. According to surface profilometry measurements, the BHJ mean thickness was $\sim 470 \mathrm{~nm}$ for samples with PEDOT:PSS IL, $\sim 700 \mathrm{~nm}$ for the SAM IL, $\sim 550 \mathrm{~nm}$ for the P3HT IL and $\sim 700 \mathrm{~nm}$ for the OPD without IL. After BHJ deposition all samples were annealed at $140^{\circ} \mathrm{C}$ for $5 \mathrm{~min}$ in inert conditions before thermal evaporation of $100 \mathrm{~nm}$ of aluminum as top electrode through a shadow mask. Diodes were encapsulated with solvent-free epoxy glue and a $100 \mu \mathrm{m}$ thick transparent glass slide. Finally, OPDs were annealed at $80^{\circ} \mathrm{C}$ for $30 \mathrm{~min}$ on a hotplate. GOS particles with $\sim 4 \mu \mathrm{m}$ average diameter were sedimented from an isopropanol dispersion onto a transparent glass resulting in a scintillator thin film with a fill factor of $\sim 33 \%$.

Light characterization. $I-V$ characteristics of the diodes were recorded using a Keithley 6487 picoammeter in an electrically and optically shielded box.

Photocurrents are measured with illumination through the transparent conductive electrode. For illumination of the photodiodes, a green light-emitting diode with a wavelength of $532 \mathrm{~nm}$ wavelength and irradiation intensity of $780 \mu \mathrm{W} \mathrm{cm}$ cm $^{-2}$ was used. EQE spectra were recorded using a lock-in technique using a chopping frequency of $170 \mathrm{~Hz}$ and an Oriel Cornerstone $1301 / 8$ m monocromator and a $\mathrm{Si}$ photodiode as reference diode for the calibration.

$\mathrm{X}$-ray characterization. $\mathrm{X}$-rays photons with energy of $70 \mathrm{keV}$ were generated with a commercial X-ray source. X-ray doses were measured with a calibrated dosimeter. Distance between organic photodiode and X-ray source was $\sim 130 \mathrm{~cm}$.

Dynamic measurements. Dynamic measurements were made in an electrically and optically shielded box with a transimpedance amplifier (FEMTO DHPCA-100) 
connected to a digital oscilloscope (LeCroy Wavesurfer 424). A low pass filter from SRS (SIM965) was inserted to reduce the high frequency noise. Through the transparent ITO electrode a green pulsed light was applied by a high power LED controlled by a signal waveform generator (TGA 1242) to create a square wave with frequencies ranging from $10 \mathrm{mHz}$ to $1 \mathrm{MHz}$. The incident light intensity was decreased from $\mathrm{mW} \mathrm{cm}^{-2}$ to $\mathrm{nW} \mathrm{cm}{ }^{-2}$ with neutral densities filters ranging from 0.1 to 4 .

Thermally stimulated current measurements. TSC measurements were performed in a closed-cycle cryostat with $\mathrm{He}$ as contact gas. Trap filling was achieved at $18 \mathrm{~K}$ with OPDs illumination for $5 \mathrm{~min}$ by a $10 \mathrm{~W}$ high power white light emitting diode (Seoul). After a dwell time of $5 \mathrm{~min}$ the temperature was increased with a constant heating rate of $7 \mathrm{~K} \mathrm{~min}^{-1}$ up to $300 \mathrm{~K}$. Thermally stimulated currents were detected by a Sub-Femtoamp Remote Source Meter (Keithley 6430). During measurements no external electric field was applied to avoid an overlap of the thermally stimulated and injected current, implying that detrapped charge carriers were extracted from the OPDs only by the built-in voltage.

1. Ng, T. N., Wong, W. S., Chabinyc, M. L., Sambandan, S. \& Street, R. A. Flexible image sensor array with bulk heterojunction organic photodiode. Appl. Phys. Lett. 92, 213303 (2008).

2. Rauch, T. et al. Near-infrared imaging with quantum-dot-sensitized organic photodiodes. Nature Photon. 3, 332-336 (2009).

3. Sariciftci, N. S., Smilowitz, L., Heeger, A. J. \& Wudl, F. Photoinduced Electron Transfer from a Conducting Polymer to Buckminsterfullerene. Science. 258, 1474-1476 (1992)

4. Tedde, S. F. et al. Fully Spray Coated Organic Photodiodes. Nano Lett. 9, 980-983 (2009).

5. Punke, M. et al. Dynamic characterization of organic bulk heterojunction photodetectors. Appl. Phys. Lett. 91, 071118 (2007).

6. Baierl, D., Fabel, B., Lugli, P. \& Scarpa, G. Efficient indium-tin-oxide (ITO) free top-absorbing organic photodetector with highly transparent polymer top electrode. Org. Electron. 12, 1669-1673 (2011).

7. Tzabari, L. \& Tessler, N. Shockley-Read-Hall recombination in P3HT:PCBM solar cells as observed under ultralow light intensities. J. Appl. Phys. 109, 064501 (2011).
8. Gao, Y. Surface Analytical Studies of Interfaces in Organic Semiconductor Devices. Materials Sci. Engr. Rep. 68, 39 (2010).

9. Schafferhans, J., Baumann, A., Deibel, C. \& Dyakonov, V. Trap distribution and the impact of oxygen-induced traps on the charge transport in poly (3-hexylthiophene). Appl. Phys. Lett. 93, 093303 (2008).

10. Schafferhans, J., Deibel, C. \& Dyakonov, V. Electronic Trap States in Methanofullerenes. Adv. Energy Mater. 1, 655-660 (2011).

11. Schafferhans, J., Baumann, A., Wagenpfahl, A., Deibel, C. \& Dyakonov, V. Oxygen doping of P3HT:PCBM blends: Influence on trap states, charge carrier mobility and solar cell performance. Org. Electron. 11, 1693-1700 (2010).

12. Agostinelli, T. et al. Trapping effects on the frequency response of dithiolenebased planar photodetectors. Synth. Met. 157, 984-987 (2007).

\section{Acknowledgments}

F.A. is recipient of an Ernst von Siemens Doctoral Fellowship (Ernst-von-Siemens-Promotionsstipendien EvS). F.A. acknowledges technical assistance of Achim Weissbeck and fruitful discussions with Reiner F. Schulz, Guenter Schmid, and

Oliver Schmidt. GOS thin films were prepared by Guenther Scholz at Siemens Healthcare.

\section{Author contributions}

F.A. carried out the experiments. J.R. performs the TSC experiments. F.A. and O.H. wrote the manuscript. All authors discussed the results and commented on the manuscript.

\section{Additional information}

Supplementary information accompanies this paper at http://www.nature.com/ scientificreports

Competing financial interests: The authors declare no competing financial interests.

License: This work is licensed under a Creative Common

Attribution-NonCommercial-NoDerivs 3.0 Unported License. To view a copy of this license, visit http://creativecommons.org/licenses/by-nc-nd/3.0/

How to cite this article: Arca, F. et al. Interface Trap States in Organic Photodiodes. Sci. Rep. 3, 1324; DOI:10.1038/srep01324 (2013). 